\title{
Lesser Toxicities of Belotecan in Patients with Small Cell Lung Cancer: A Retrospective Single-Center Study of Camptothecin Analogs
}

\author{
Yeon Hee Park, ${ }^{1}$ Chae Uk Chung, ${ }^{1}$ Bo Mi Park, ${ }^{2}$ Myoung Rin Park, \\ Dong Il Park, ${ }^{1}$ Jae Young Moon, ${ }^{1}$ Hee Sun Park, ${ }^{1}$ Jin Hwan Kim, ${ }^{3}$ \\ Sung Soo Jung, ${ }^{1}$ Ju Ock Kim, ${ }^{1}$ Sun Young Kim, ${ }^{1}$ and Jeong Eun Lee ${ }^{1}$ \\ ${ }^{1}$ Department of Internal Medicine, College of Medicine, Chungnam National University, Daejeon, Republic of Korea \\ ${ }^{2}$ Department of Internal Medicine, College of Medicine, Eulji University, Daejeon, Republic of Korea \\ ${ }^{3}$ Department of Radiology, College of Medicine, Chungnam National University, Daejeon, Republic of Korea
}

Correspondence should be addressed to Jeong Eun Lee; vov-x@daum.net

Received 18 July 2016; Revised 6 October 2016; Accepted 23 October 2016

Academic Editor: Hisao Imai

Copyright (C) 2016 Yeon Hee Park et al. This is an open access article distributed under the Creative Commons Attribution License, which permits unrestricted use, distribution, and reproduction in any medium, provided the original work is properly cited.

Purpose. Topotecan and belotecan are camptothecin derivatives that are used to treat small cell lung cancer (SCLC). This study compared the toxicities and efficacies of belotecan and topotecan monotherapies in patients with SCLC. Methods. We retrospectively reviewed data from 94 patients with SCLC (with or without prior chemotherapy) who were treated using belotecan monotherapy ( $n=59,188$ cycles) or topotecan monotherapy $(n=35,65$ cycles) between September 2003 and December 2011. Results. Thrombocytopenia occurred during $42 \%$ and $61.5 \%$ of the belotecan and topotecan cycles, respectively $(p=0.007)$. Significant differences between belotecan and topotecan were also observed for grade $4 / 5$ lung infection ( $3.2 \%$ versus $10.8 \%$, resp.; $p=0.003$ ), all-grade headache (3.2\% versus $10.8 \%$, resp.; $p=0.017)$, and grade $4 / 5$ increased liver enzymes ( $0.5 \%$ versus $4.6 \%$, resp.; $p=0.023)$. The median TTPDs, CSSs, and OSs were 14 months and 11.6 months $(p=0.646), 10$ months and 7 months $(p=0.179)$, and 34.5 months and 21.4 months ( $p=0.914$ ) after belotecan and topotecan monotherapy, respectively. Conclusions. Belotecan monotherapy may be safer than topotecan monotherapy in SCLC patients. And in terms of efficacy, belotecan could be comparable to topotecan monotherapy.

\section{Introduction}

Small cell lung cancer (SCLC) accounts for approximately $15 \%$ of all lung cancer cases in United States [1-3]. And in South Korea, it also accounts for $12.5 \%$ of lung cancer in 2012 $[4,5]$. Unfortunately, treatment is not effective in altering the high recurrence rate and short survival, regardless of the initial stage of SCLC at the diagnosis [6, 7]. Therefore, combination chemotherapy (e.g., etoposide/cisplatin) remains the main treatment for patients with extensive-stage SCLC [8-12]. The etoposide/cisplatin combination provides initial response rates of up to $80 \%$, although most patients ultimately relapse and receive second-line therapy $[13,14]$. However, there are only a few chemotherapeutic drugs that can be used as second-line therapy for SCLC. Various single-agent treatments (e.g., topotecan, belotecan, etoposide, irinotecan, gemcitabine, and pemetrexed) have been approved as secondline chemotherapy for patients with SCLC. The response rates for single-agent treatments are $0-47 \%$ [15]. As there are few effective treatments for SCLC, additional safe and effective treatments are urgently needed.

Topotecan is approved as chemotherapeutic drug for relapsed or failed after first-line therapy of SCLC. It is also available as intravenous and oral agent. Topotecan showed greater response rate than combination of cyclophosphamide, doxorubicin, and vincristine regimen, although median survival was similar [16]. In a randomized controlled trial, oral topotecan was superior to best supportive care in improving survival 
and quality of life [17]. As second-line therapy, topotecan demonstrated response rate of $10 \sim 40 \%$ and median survival time of 6.0 months [18].

Belotecan (Camtobell ${ }^{\circledR}$, CKD602, 7-[2-(N-isopropylamino)ethyl]-(20S)-camptothecin, Chong Keun Dang Corp.) is a novel camptothecin derivative that has a water-solubilizing chemical group attached to the B ring. There are few studies that have compared the clinical effects of belotecan and topotecan monotherapies in patients with SCLC. Therefore, this study aimed to assess and compare toxicities and efficacies between belotecan and topotecan monotherapies in patients with SCLC.

\section{Patients and Methods}

This retrospective study evaluated patients with SCLC who had received belotecan or topotecan monotherapy. The study's design was approved by the institutional review board of Chungnam National University Hospital (2013-01-008). All patients were informed that they were eligible to receive belotecan or topotecan monotherapy, and their consent to receive treatment was recorded in their medical records.

2.1. Eligibility Criteria. All data were derived from a database of patients who were diagnosed with SCLC between September 2003 and December 2011. The inclusion criteria were histological or cytological confirmation of SCLC, treatment with belotecan or topotecan monotherapy for SCLC (firstline, second-line, or third-line), no prior chemotherapy using an topoisomerase I inhibitor, no other malignant disease, no uncontrolled disease that might have affected the patient's survival, and no chemotherapy within 3 weeks of the study period. The laboratory criteria were white blood cell counts of $\geq 3,000 / \mathrm{mm}^{3}$, absolute neutrophil counts of $\geq 1,000 / \mathrm{mm}^{3}$, platelet counts of $\geq 100,000 / \mathrm{mm}^{3}$, hemoglobin levels of $\geq 10.0 \mathrm{~g} / \mathrm{dL}$, serum bilirubin levels of $\leq 1.8 \mathrm{mg} / \mathrm{dL}$, serum transaminase levels of $\leq 100 \mathrm{IU} / \mathrm{L}$, and serum creatinine levels of $\leq 1.5 \mathrm{mg} / \mathrm{dL}$.

2.2. Chemotherapy. All patients in the present study received belotecan or topotecan monotherapy as first-line, secondline, or third-line therapy. All patients were required to wait at least 3 weeks after their last cycle before undergoing secondor third-line therapy. Belotecan monotherapy was administered at $0.5 \mathrm{mg} / \mathrm{m}^{2} /$ day for 5 consecutive days every 3 weeks. The belotecan was mixed with $100 \mathrm{~mL}$ of $5 \%$ dextrose and administered as a $30 \mathrm{~min}$ intravenous injection. Topotecan monotherapy was administered at $1.5 \mathrm{mg} / \mathrm{m}^{2} /$ day for 5 consecutive days every 3 weeks. The topotecan was mixed with $200 \mathrm{~mL}$ of $5 \%$ dextrose and administered as a $30 \mathrm{~min}$ intravenous injection. Dose adjustments were made at the start of each new cycle and were made based on the worst toxicity that was observed during the previous cycle. If the patient experienced grade $4 / 5$ toxicities, the topotecan dose reduction was $0.25 \mathrm{mg} / \mathrm{m}^{2}$ or a $20 \%$ reduction from the previous dose of belotecan. Belotecan or topotecan monotherapy was terminated when the treatment response revealed progressive disease or chemotherapy-induced toxicity that was uncontrolled by consecutive dose reductions or if the treatment schedule was delayed by $>2$ weeks.

2.3. Response and Toxicity Evaluation. Tumor response was evaluated using the Response Evaluation Criteria in Solid Tumours (RECIST, version 1.1) via enhanced computed tomography. Hematological and nonhematological toxicities were evaluated using the Common Terminology Criteria for Adverse Events (version 4.0).

2.4. Survival Analysis. Time to progressive disease (TTPD) was defined as the time from the beginning of belotecan or topotecan monotherapy to the date of diagnosing radiologically confirmed progressive disease (PD). Chemotherapyspecific survival (CSS) was defined as the time from the beginning of belotecan or topotecan monotherapy to the date of cancer-related death or the end of the study. Overall survival (OS) was defined as the time from the date of histological or pathological confirmation to the date of cancer-related death or the end of the study.

2.5. Statistical Analysis. We used the chi-square and independent $t$-tests to analyze the differences in the patients' baseline characteristics and toxicities. We also used the chi-square test and Fisher's exact test to analyze the differences in toxicities between patients who were $<75$ years old and $\geq 75$ years old. The TTPD, CSS, and OS values for the two chemotherapy groups were evaluated using the Kaplan-Meier method and the log-rank test. All statistical analyses were performed using SPSS software (version 19; SPSS Inc., Chicago, IL), and a $p$ value of $<0.05$ was considered statistically significant.

\section{Results}

3.1. Baseline Characteristics of the Two Treatment Groups. Between September 2003 and November 2011, we identified 94 patients who have received topotecan monotherapy $(n=$ $35)$ or belotecan monotherapy $(n=59)$ (Table 1$)$. The median patient age was 68 years (range, $42-88$ years), and there were no significant differences between the two treatment groups, except in their previous lines of chemotherapy. Most treatments for all patients were second-line $(60.6 \%)$ and third-line (25.5\%) therapy, respectively. Most patients in the belotecan group received belotecan as second-line therapy (67.8\%). In topotecan group, the proportion of patients who received as second-line was $48.6 \%$ and as third-line was $42.9 \%$.

3.2. Tumor Response. Among the 94 included patients, 28 patients could not be evaluated for response, due to inadequate radiological data (Table 2). Fifteen of these unevaluable patients were treated using topotecan and 13 patients were treated using belotecan. Among the 46 evaluable patients who received belotecan, the best overall responses were complete response (CR) in 2 patients (4.3\%), partial response (PR) in 8 patients (17.4\%), stable disease (SD) in 19 patients $(41.3 \%)$, and $\mathrm{PD}$ in 17 patients (37\%). The overall response rate (ORR) for belotecan was $21.7 \%$ in the intent-to-treat analysis. Among the 20 evaluable patients who received topotecan, the best 
TABLE 1: Baseline characteristics.

\begin{tabular}{|c|c|c|c|c|c|c|c|}
\hline \multirow{2}{*}{ Parameter } & \multicolumn{2}{|c|}{ All patients $(n=94)$} & \multicolumn{2}{|c|}{ Belotecan group $(n=59)$} & \multicolumn{2}{|c|}{ Topotecan group $(n=35)$} & \multirow{2}{*}{$p$ value } \\
\hline & Number of patients & $\%$ & Number of patients & $\%$ & Number of patients & $\%$ & \\
\hline Sex & & & & & & & 0.054 \\
\hline Male & 80 & 85.1 & 47 & 79.7 & 33 & 94.3 & \\
\hline Female & 14 & 14.9 & 12 & 20.3 & 2 & 5.7 & \\
\hline Age, years & & & & & & & 0.164 \\
\hline Median & 67.8 & & 68.8 & & 65.8 & & \\
\hline Range & $42-88$ & & $42-88$ & & $48-81$ & & \\
\hline$E C O G$ & & & & & & & 0.715 \\
\hline 0 & 17 & 18.1 & 10 & 16.9 & 7 & 20 & \\
\hline 1 & 48 & 51.1 & 31 & 52.5 & 17 & 48.6 & \\
\hline 2 & 18 & 19.1 & 11 & 18.6 & 7 & 20 & \\
\hline 3 & 10 & 10.6 & 7 & 11.9 & 3 & 8.6 & \\
\hline 4 & 1 & 1.1 & 0 & 0 & 1 & 2.9 & \\
\hline Stage at diagnosis & & & & & & & 0.330 \\
\hline Limited & 44 & 46.8 & 32 & 54.2 & 12 & 34.3 & \\
\hline IIa & 2 & 2.1 & 2 & 3.4 & 0 & 0 & \\
\hline IIb & 1 & 1.1 & 1 & 1.7 & 0 & 0 & \\
\hline IIIa & 15 & 16 & 11 & 18.6 & 4 & 11.4 & \\
\hline IIIB & 26 & 27.7 & 18 & 30.5 & 8 & 22.9 & \\
\hline Extensive & 50 & 53.2 & 27 & 45.8 & 23 & 65.7 & \\
\hline Response to prior CTx & & & & & & & 0.088 \\
\hline CR or PR & 11 & 11.7 & 10 & 16.9 & 1 & 2.9 & \\
\hline $\mathrm{SD}$ or $\mathrm{PD}$ & 55 & 58.5 & 36 & 61 & 19 & 54.3 & \\
\hline CTx line & & & & & & & 0.011 \\
\hline 1 & 13 & 13.8 & 10 & 16.9 & 3 & 8.6 & \\
\hline 2 & 57 & 60.6 & 40 & 67.8 & 17 & 48.6 & \\
\hline 3 & 24 & 25.5 & 9 & 15.3 & 15 & 42.9 & \\
\hline
\end{tabular}

ECOG: Eastern Cooperative Oncology Group score, CTx: chemotherapy, CR: complete response, PR: partial response, SD: stable disease, and PD: progressive disease.

TABLE 2: Chemotherapy response.

\begin{tabular}{lcccccc}
\hline & \multicolumn{2}{c}{$\begin{array}{c}\text { All patients } \\
(n=94)\end{array}$} & \multicolumn{2}{c}{$\begin{array}{c}\text { Belotecan } \\
(n=59)\end{array}$} & \multicolumn{2}{c}{$\begin{array}{c}\text { Topotecan } \\
(n=35)\end{array}$} \\
& Number & $\%$ & Number & $\%$ & Number & $\%$ \\
\hline $\begin{array}{l}\text { Complete } \\
\text { response }\end{array}$ & $2 / 66$ & 3.0 & $2 / 46$ & 4.3 & 0 & 0 \\
$\begin{array}{l}\text { Partial } \\
\text { response }\end{array}$ & $9 / 66$ & 13.6 & $8 / 46$ & 17.4 & $1 / 20$ & 5 \\
$\begin{array}{l}\text { Stable disease } \\
\begin{array}{l}\text { Progressive } \\
\text { disease }\end{array}\end{array}$ & $26 / 66$ & 26.3 & $19 / 46$ & 41.3 & $7 / 20$ & 35 \\
$\begin{array}{l}\text { Not evaluated } \\
\text { Response rate }\end{array}$ & $28 / 94$ & 43.9 & $17 / 46$ & 37 & $12 / 20$ & 60 \\
\hline
\end{tabular}

Response rate $=$ complete response + partial response.

overall responses were $\mathrm{PR}$ in 1 patient (5\%), SD in 7 patients (35\%), and PD in 12 patients (60\%). The ORR in the topotecan group was $5 \%$, which was noticeably lower than that in the belotecan group.
3.3. Toxicity. The toxicity analyses for each group were based on the total number of treatment cycles. The belotecan group contained 59 patients who underwent 188 cycles (mean 3.19 cycles), and the topotecan group contained 35 patients who underwent 65 cycles (mean 1.86 cycles) (Table 3). Among the hematological toxicities, all-grade thrombocytopenia and grade 4/5 thrombocytopenia were significantly more common in the topotecan group, compared to the belotecan group ( $p=0.007$ and $p=0.001$, resp.). There were no statistically significant differences in the frequencies of anemia and neutropenia, although all-grade anemia was slightly more frequent in the belotecan group, and all-grade neutropenia was slightly more frequent in the topotecan group. We also evaluated a wide variety of nonhematological toxicities. Compared to the belotecan group, the topotecan group exhibited significantly more frequent grade $4 / 5$ lung infection ( $p=$ $0.003)$, grade $4 / 5$ increased liver enzymes $(p=0.23)$, and allgrade headache $(p=0.017)$.

3.4. Comparing Toxicities according to Age. The toxicities were also compared for patients who were $<75$ years old and 
TABle 3: Toxicities (CTCAE version 4.0).

\begin{tabular}{|c|c|c|c|c|c|c|c|c|c|c|}
\hline \multirow{3}{*}{ CTCAE toxicity } & \multicolumn{4}{|c|}{ Belotecan $(n=188)$} & \multicolumn{4}{|c|}{ Topotecan $(n=65)$} & \multirow{3}{*}{$\begin{array}{c}\text { All grades } \\
p \text { value }\end{array}$} & \multirow{3}{*}{$\begin{array}{c}\text { Grade } 4 / 5 \\
p \text { value }\end{array}$} \\
\hline & \multicolumn{2}{|c|}{ All grades } & \multicolumn{2}{|c|}{ Grade 4/5 } & \multicolumn{2}{|c|}{ All grades } & \multicolumn{2}{|c|}{ Grade 4/5 } & & \\
\hline & Number & $\%$ & Number & $\%$ & Number & $\%$ & Number & $\%$ & & \\
\hline \multicolumn{11}{|l|}{ Hematological toxicity } \\
\hline Anemia & 125 & 66.5 & 3 & 1.6 & 42 & 64.6 & 0 & 0 & 0.783 & 0.306 \\
\hline Neutropenia & 120 & 63.8 & 56 & 29.8 & 47 & 72.3 & 24 & 36.9 & 0.214 & 0.286 \\
\hline Thrombocytopenia & 79 & 42 & 12 & 6.4 & 40 & 61.5 & 14 & 21.5 & 0.007 & 0.001 \\
\hline \multicolumn{11}{|l|}{ Nonhematological toxicity } \\
\hline Prurigo nodularis & 0 & 0 & 0 & 0 & 1 & 1.5 & 0 & 0 & 0.088 & \\
\hline Generalized muscle weakness & 22 & 11.7 & 0 & 0 & 10 & 15.4 & 0 & 0 & 0.441 & \\
\hline Anorexia & 24 & 12.8 & 0 & 0 & 9 & 13.8 & 0 & 0 & 0.824 & \\
\hline Constipation & 7 & 3.7 & 0 & 0 & 6 & 9.2 & 0 & 0 & 0.083 & \\
\hline Nausea & 15 & 8 & 0 & 0 & 5 & 7.7 & 0 & 0 & 0.941 & \\
\hline Vomiting & 5 & 2.7 & 0 & 0 & 2 & 3.1 & 0 & 0 & 0.860 & \\
\hline Dyspnea & 4 & 2.1 & 0 & 0 & 4 & 6.2 & 1 & 1.5 & 0.110 & 0.088 \\
\hline Lung infection & 12 & 6.4 & 4 & 2.1 & 9 & 13.8 & 7 & 10.8 & 0.060 & 0.003 \\
\hline Dizziness & 6 & 3.2 & 0 & 0 & 5 & 7.7 & 0 & 0 & 0.125 & \\
\hline Myalgia & 4 & 2.1 & 0 & 0 & 0 & 0 & 0 & 0 & 0.236 & \\
\hline Sinus tachycardia & 0 & 0 & 0 & 0 & 1 & 1.5 & 0 & 0 & 0.088 & \\
\hline Headache & 6 & 3.2 & 0 & 0 & 7 & 10.8 & 0 & 0 & 0.017 & \\
\hline Cough & 1 & 0.5 & 0 & 0 & 0 & 0 & 0 & 0 & 0.556 & \\
\hline Diarrhea & 8 & 4.3 & 0 & 0 & 6 & 9.2 & 0 & 0 & 0.130 & \\
\hline Skin rash & 5 & 2.7 & 0 & 0 & 2 & 3.1 & 0 & 0 & 0.860 & \\
\hline Intracranial hemorrhage & 1 & 0.5 & 1 & 0.5 & 0 & 0 & 0 & 0 & 0.556 & 0.556 \\
\hline TB increased & 0 & 0 & 0 & 0 & 1 & 1.5 & 0 & 0 & 0.088 & \\
\hline AST/ALT increased & 3 & 1.6 & 1 & 0.5 & 3 & 4.6 & 3 & 4.6 & 0.168 & 0.023 \\
\hline Abdominal pain & 3 & 1.6 & 0 & 0 & 1 & 1.5 & 0 & 0 & 0.975 & \\
\hline Hiccups & 7 & 3.7 & 0 & 0 & 1 & 1.5 & 0 & 0 & 0.386 & \\
\hline Fever & 7 & 3.7 & 0 & 0 & 1 & 1.5 & 0 & 0 & 0.386 & \\
\hline Confusion & 0 & 0 & 0 & 0 & 1 & 1.5 & 0 & 0 & 0.088 & \\
\hline Hyponatremia & 3 & 1.6 & 1 & 0.5 & 2 & 3.1 & 1 & 1.5 & 0.460 & 0.430 \\
\hline Delirium & 0 & 0 & 0 & 0 & 1 & 1.5 & 0 & 0 & 0.088 & \\
\hline Insomnia & 2 & 1.1 & 0 & 0 & 0 & 0 & 0 & 0 & 0.404 & \\
\hline Tremor & 1 & 0.5 & 0 & 0 & 0 & 0 & 0 & 0 & 0.556 & \\
\hline Sore throat & 3 & 1.6 & 0 & 0 & 0 & 0 & 0 & 0 & 0.306 & \\
\hline URI & 1 & 0.5 & 0 & 0 & 0 & 0 & 0 & 0 & 0.556 & \\
\hline Viral hepatitis & 1 & 0.5 & 1 & 0.5 & 0 & 0 & 0 & 0 & 0.556 & 0.556 \\
\hline Dyspepsia & 0 & 0 & 0 & 0 & 1 & 1.5 & 0 & 0 & 0.088 & \\
\hline Ileus & 0 & 0 & 0 & 0 & 1 & 1.5 & 0 & 0 & 0.088 & \\
\hline Atrial fibrillation & 1 & 0.5 & 0 & 0 & 0 & 0 & 0 & 0 & 0.556 & \\
\hline Hypokalemia & 1 & 0.5 & 0 & 0 & 0 & 0 & 0 & 0 & 0.556 & \\
\hline Pleural infection & 1 & 0.5 & 0 & 0 & 1 & 1.5 & 0 & 0 & 0.430 & \\
\hline
\end{tabular}

CTCAE: Common Terminology Criteria for Adverse Events, TB: total bilirubin, ALT: alanine transaminase, AST: aspartate transaminase, and URI: upper respiratory tract infection. 
TABLE 4: Comparing the toxicities for patients who were $<75$ years or $\geq 75$ years old.

\begin{tabular}{|c|c|c|c|c|c|c|c|c|c|c|}
\hline \multirow{3}{*}{ CTCAE terminology } & \multicolumn{4}{|c|}{ Belotecan (188 cycles) } & \multicolumn{6}{|c|}{ Topotecan (65 cycles) } \\
\hline & \multicolumn{2}{|c|}{$\begin{array}{l}<75 \text { years } \\
(124 \text { cycles })\end{array}$} & \multicolumn{2}{|c|}{$\begin{array}{l}\geq 75 \text { years } \\
(64 \text { cycles })\end{array}$} & \multirow[t]{2}{*}{$p$ value } & \multicolumn{2}{|c|}{$\begin{array}{l}<75 \text { years } \\
(53 \text { cycles })\end{array}$} & \multicolumn{2}{|c|}{$\begin{array}{l}\geq 75 \text { years } \\
\text { (12 cycles) }\end{array}$} & \multirow[t]{2}{*}{$p$ value } \\
\hline & Number & $\%$ & Number & $\%$ & & Number & $\%$ & Number & $\%$ & \\
\hline \multicolumn{11}{|l|}{ Hematological toxicity } \\
\hline Anemia & 82 & 66.1 & 42 & 65.6 & 0.945 & 35 & 66 & 7 & 58.3 & 0.741 \\
\hline Neutropenia & 72 & 58.1 & 49 & 76.6 & 0.012 & 38 & 71.7 & 9 & 75 & 1.000 \\
\hline Thrombocytopenia & 55 & 44.4 & 23 & 35.9 & 0.267 & 33 & 62.3 & 7 & 58.3 & 1.000 \\
\hline \multicolumn{11}{|l|}{ Nonhematological toxicity } \\
\hline Generalized muscle weakness & 11 & 8.9 & 11 & 17.2 & 0.093 & 10 & 18.9 & 0 & 0 & \\
\hline Anorexia & 15 & 12.1 & 9 & 14.1 & 0.702 & 9 & 17 & 0 & 0 & \\
\hline Constipation & 2 & 1.6 & 5 & 7.8 & 0.046 & 4 & 7.5 & 2 & 16.7 & 0.305 \\
\hline Nausea & 12 & 9.7 & 3 & 4.7 & 0.232 & 5 & 9.4 & 0 & 0 & \\
\hline Vomiting & 4 & 3.2 & 1 & 1.6 & 0.663 & 2 & 3.8 & 0 & 0 & \\
\hline Dyspnea & 1 & 0.8 & 3 & 4.7 & 0.115 & 3 & 5.7 & 0 & 0 & \\
\hline Lung infection & 8 & 6.5 & 3 & 4.7 & 0.752 & 7 & 13.2 & 2 & 16.7 & 0.667 \\
\hline Dizziness & 5 & 4.0 & 1 & 1.6 & 0.666 & 5 & 9.4 & 0 & 0 & \\
\hline Myalgia & 2 & 1.6 & 2 & 3.1 & 0.606 & 0 & 0 & 0 & 0 & \\
\hline Sinus tachycardia & 0 & 0 & 0 & 0 & & 1 & 1.9 & 0 & 0 & \\
\hline Headache & 6 & 4.8 & 0 & 0 & & 7 & 13.2 & 0 & 0 & \\
\hline Cough & 1 & 0.8 & 0 & 0 & & 0 & 0 & 0 & 0 & \\
\hline Diarrhea & 3 & 2.4 & 5 & 7.8 & 0.124 & 3 & 5.7 & 3 & 25 & 0.071 \\
\hline Skin rash & 1 & 0.8 & 4 & 6.3 & 0.047 & 2 & 3.8 & 0 & 0 & \\
\hline Intracranial hemorrhage & 1 & 0.8 & 0 & 0 & & 0 & 0 & 0 & 0 & \\
\hline TB increased & 0 & 0 & 0 & 0 & & 1 & 1.9 & 0 & 0 & \\
\hline AST/ALT increased & 2 & 1.6 & 1 & 1.6 & 1.000 & 3 & 5.7 & 0 & 0 & \\
\hline Abdominal pain & 3 & 2.4 & 0 & 0 & & 1 & 1.9 & 0 & 0 & \\
\hline Hiccups & 6 & 4.8 & 1 & 1.6 & 0.426 & 1 & 1.9 & 0 & 0 & \\
\hline Fever & 5 & 4.0 & 2 & 3.1 & 1.000 & 1 & 1.9 & 0 & 0 & \\
\hline Confusion & 0 & 0 & 0 & 0 & & 1 & 1.9 & 0 & 0 & \\
\hline Hyponatremia & 3 & 2.4 & 0 & 0 & & 2 & 3.8 & 0 & 0 & \\
\hline Delirium & 0 & 0 & 0 & 0 & & 1 & 1.9 & 0 & 0 & \\
\hline Insomnia & 2 & 1.6 & 0 & 0 & & 0 & 0 & 0 & 0 & \\
\hline Tremor & 1 & 0.8 & 0 & 0 & & 0 & 0 & 0 & 0 & \\
\hline Sore throat & 2 & 1.6 & 1 & 1.6 & 1.000 & 0 & 0 & 0 & 0 & \\
\hline URI & 0 & 0 & 1 & 1.6 & & 0 & 0 & 0 & 0 & \\
\hline Viral hepatitis & 0 & 0 & 1 & 1.6 & & 0 & 0 & 0 & 0 & \\
\hline Dyspepsia & 0 & 0 & 0 & 0 & & 1 & 1.9 & 0 & 0 & \\
\hline Ileus & 0 & 0 & 0 & 0 & & 1 & 1.9 & 0 & 0 & \\
\hline Atrial fibrillation & 1 & 0.8 & 0 & 0 & & 0 & 0 & 0 & 0 & \\
\hline Hypokalemia & 1 & 0.8 & 0 & 0 & & 0 & 0 & 0 & 0 & \\
\hline Pleural infection & 1 & 0.8 & 0 & 0 & & 1 & 1.9 & 0 & 0 & \\
\hline
\end{tabular}

CTCAE: Common Terminology Criteria for Adverse Events, TB: total bilirubin, ALT: alanine transaminase, AST: aspartate transaminase, and URI: upper respiratory tract infection.

$\geq 75$ years old (Table 4). Among patients who received belotecan monotherapy, neutropenia was significantly more frequent for patients who were $\geq 75$ years old ( $77 \%$ versus $58 \%$, $p=0.012$ ). Among the nonhematological toxicities, patients who were $\geq 75$ years old exhibited significantly higher frequencies of skin rash $(6.3 \%$ versus $0.8 \%, p=0.047)$ and constipation $(7.8 \%$ versus $1.6 \%, p=0.046)$. However, there were no age-related differences in the toxicities among the patients who received topotecan monotherapy.

3.5. Survival Analysis. For the belotecan group, the median TTPD was 14.0 months, the median CSS was 10.0 months, and 
the median OS was 34.5 months. For the topotecan group, the median TTPD was 11.6 months, the median CSS was 7.0 months, and the median OS was 21.4 months (see Supplemental Figure 1 available online at http://dx.doi.org/10.1155/ 2016/3576201). There were no significant differences in TTPD, CSS, and OS between the two treatment groups $(p=0.646$, $p=0.179$, and $p=0.914$, resp.).

\section{Discussion}

In the present study, we observed that belotecan monotherapy and topotecan monotherapy had comparable efficacies in SCLC. However, compared to topotecan monotherapy, belotecan monotherapy was associated with significantly less frequent thrombocytopenia (all-grade and grade 4/5), grade 4/5 lung infection, and all-grade headache. Therefore, we cautiously speculate that belotecan may be preferable to topotecan, given the similar survival outcomes and superior safety profile of belotecan.

In a tumor xenograft model, belotecan provided an approximately 3-fold more potent antitumor effect, compared to topotecan. Furthermore, the therapeutic margin for belotecan was 4-fold higher than that for topotecan [19]. In other words, belotecan could be more safely used than topotecan.

Topotecan and belotecan monotherapies are both second-line options for SCLC; however, only two studies had compared the survival rates and toxicities of topotecan and belotecan in patients with SCLC and both were reported as abstracts [20]. Yoon et al. compared belotecan and topotecan as second-line treatment in SCLC patients. ORR, PFS, and OS of belotecan and topotecan were not significant different. However, grade $3 / 4$ neutropenia was more common in topotecan group (43.6\%) than belotecan group $(21.3 \%)$ ( $p=$ 0.016). A prospective phase III study comparing belotecan and topotecan was also reported at the 112th Annual Meeting of Korean Academy of Tuberculosis and Respiratory Diseases (in 2011) [21]. They compared the efficacies and hematological toxicities of belotecan and topotecan in 54 patients with relapsed SCLC after receiving platinum/etoposide combination therapy. Compared to belotecan monotherapy, topotecan monotherapy was associated with more frequent grade $3 / 4$ hematological toxicity (64.3\% versus $96.2 \%$, resp.), although the two groups exhibited similar efficacy outcomes (including ORR, disease control rate, OS, and progression-free survival). Those findings are also consistent with the findings of the present study.

We compared two phase II trials of belotecan and topotecan which included previously untreated extensive-stage SCLC $[22,23]$. Belotecan study showed superior efficacy in ORR (53.2\% versus $39.0 \%)$ and 1 year-survival rate $(49.9 \%$ versus $39 \%$ ), although TTPD and OS were similar in both studies. Interestingly, grade $3 / 4$ neutropenia and thrombocytopenia were $71.0 \%$ and $12.9 \%$ in belotecan study. However, in topotecan study, grade $3 / 4$ neutropenia and thrombocytopenia were $92 \%$ and $38 \%$. These data also support our result that belotecan therapy was better in safety profile than topotecan therapy.
Another study compared the efficacies and toxicities of topotecan- and belotecan-based chemotherapies for recurrent epithelial ovarian cancer. In that study, belotecan monotherapy provided a superior safety profiles compared to topotecan monotherapy. Grade 3/4 anemia and thrombocytopenia were less frequent during belotecan-based chemotherapy [24].

In this study, belotecan had greater response rate and survival rate than topotecan. However, it was not statistically significant. These results would be influenced by different proportion of patients between two groups. In topotecan group, patients who were treated as third line were $42.9 \%$; however, $15.3 \%$ of belotecan group were treated as third line. Furthermore, topotecan group had been performed fewer cycles than belotecan group (mean 1.86 versus 3.19 cycles). This also would have impact on efficacy outcome.

Moreover, older patient group (who were $\geq 75$ years old) had more adverse events than younger patient group (who were $<75$ years old) when treated by belotecan. Therefore, we should be cautious when treating elderly patients with belotecan therapy.

4.1. Limitations. The first limitation of the present study is its retrospective design. The second limitation is the fact that we cannot account for the effects of previous treatment(s). For example, belotecan was most frequently used as a second-line therapy ( $n=40,67.8 \%)$, which was followed by first-line therapy ( $n=10,16.9 \%)$. Topotecan was also most frequently used as a second-line therapy $(n=17,48.6 \%)$, although this was followed by third-line therapy ( $n=15,42.9 \%)$, and these differences were statistically significant. Therefore, we cannot overlook the possible effects of the previous chemotherapeutic regimens. Nevertheless, all subsequent lines of therapy were delayed until the patients' symptoms had improved and their laboratory findings were normal, which may have prevented any cumulative toxicity. The third limitation is that we could not clearly distinguish the nonhematological toxicities (e.g., headache and dyspnea) from the symptoms of lung cancer. The fourth limitation is that response for $29.8 \%$ of all patients were not evaluated. For that reason, we could not compare survival and response rate precisely. Thus, further prospective studies are needed to evaluate the efficacies and toxicities of belotecan and topotecan monotherapies, in order to develop more potent and well-tolerated chemotherapies.

\section{Conclusion}

In the present study, hematological toxicities were generally more frequent among patients who received topotecan monotherapy, and a significant difference was observed for grade 4/5 thrombocytopenia. A similar trend was observed for nonhematological toxicities, with significant differences being observed for all-grade headache, grade $4 / 5$ increased liver enzymes, and grade $4 / 5$ lung infection. Therefore, based on our experience, belotecan monotherapy may be safer than topotecan monotherapy in SCLC and it also showed comparable efficacies. 


\author{
Abbreviations \\ SCLC: Small cell lung cancer \\ TTPD: Time to progressive disease \\ CSS: Chemotherapy-specific survival \\ PD: $\quad$ Progressive disease \\ OS: Overall survival \\ CR: Complete response \\ PR: Partial response \\ SD: $\quad$ Stable disease \\ ORR: Overall response rate.
}

\section{Ethical Approval}

All procedures performed in studies involving human participants were in accordance with the ethical standards of the institutional research committee and with the 1964 Helsinki declaration and its later amendments or comparable ethical standards. This study was approved by the Institutional Review Board of the Chungnam National University Hospital (IRB no. 2013-01-008).

\section{Competing Interests}

The authors declare that they have no conflict of interests.

\section{Authors' Contributions}

(I) Conception and design were performed by all authors. (II) Collection and assembly of data were performed by Yeon Hee Park, Chae Uk Chung, Myoung Rin Park, and Bo Mi Park. (III) Data analysis and interpretation were performed by Jeong Eun Lee, Chae Uk Chung, and Yeon Hee Park. (IV) Manuscript writing was performed by all authors. (V) Final approval of manuscript was performed by all authors. Yeon Hee Park and Chae Uk Chung contributed equally to this work as co-first author.

\section{References}

[1] R. Govindan, N. Page, D. Morgensztern et al., "Changing epidemiology of small-cell lung cancer in the United States over the last 30 years: analysis of the surveillance, epidemiologic, and end results database," Journal of Clinical Oncology, vol. 24, no. 28, pp. 4539-4544, 2006.

[2] G. R. Simon and H. Wagner, "Small cell lung cancer," Chest, vol. 123, no. 1, pp. 259S-271S, 2003.

[3] D. M. Jackman and B. E. Johnson, "Small-cell lung cancer," The Lancet, vol. 366, no. 9494, pp. 1385-1396, 2005.

[4] J. Y. Park and S. H. Jang, "Epidemiology of lung cancer in Korea: recent trends," Tuberculosis and Respiratory Diseases, vol. 79, no. 2, pp. 58-69, 2016.

[5] K. C. C. Registry, Annual Report of Cancer Statistics in Korea in 2012, 2015, http://www.cancer.go.kr/.

[6] C. L. Hann and C. M. Rudin, "Management of small-cell lung cancer: incremental changes but hope for the future," Oncology, vol. 22, no. 13, pp. 1486-1492, 2008.

[7] M. Sorensen, M. Pijls-Johannesma, E. Felip, and E. G. W. Group, "Small-cell lung cancer: ESMO Clinical Practice Guidelines for diagnosis, treatment and follow-up," Annals of Oncology, vol. 21, supplement 5, pp. v120-v125, 2010.

[8] A. Kallianos, A. Rapti, P. Zarogoulidis et al., "Therapeutic procedure in small cell lung cancer," Journal of Thoracic Disease, vol. 5, supplement 4, pp. S420-S424, 2013.

[9] K. K. Ciombor and C. M. S. Rocha Lima, "Management of small cell lung cancer," Current Treatment Options in Oncology, vol. 7, no. 1, pp. 59-68, 2006.

[10] J. Seidenfeld, D. J. Samson, C. J. Bonnell, K. M. Ziegler, and N. Aronson, "Management of small cell lung cancer," Evidence Report/Technology Assessment, pp. 1-154, 2006.

[11] J. W. Neal, M. A. Gubens, and H. A. Wakelee, "Current management of small cell lung cancer," Clinics in Chest Medicine, vol. 32, no. 4, pp. 853-863, 2011.

[12] J. R. Jett, S. E. Schild, K. A. Kesler, and G. P. Kalemkerian, "Treatment of small cell lung cancer: diagnosis and management of lung cancer, 3rd ed: American college of chest physicians evidence-based clinical practice guidelines," Chest, vol. 143, no. 5, pp. e400-e419, 2013.

[13] K. Noda, Y. Nishiwaki, M. Kawahara et al., "Irinotecan plus cisplatin compared with etoposide plus cisplatin for extensive small-cell lung cancer," The New England Journal of Medicine, vol. 346, no. 2, pp. 85-91, 2002.

[14] S. Sundstrøm, R. M. Bremnes, S. Kaasa et al., "Cisplatin and etoposide regimen is superior to cyclophosphamide, epirubicin, and vincristine regimen in small-cell lung cancer: results from a randomized phase III trial with 5 years' follow-up," Journal of Clinical Oncology, vol. 20, no. 24, pp. 4665-4672, 2002.

[15] Y. H. Kim and M. Mishima, "Second-line chemotherapy for small-Cell Lung Cancer (SCLC)," Cancer Treatment Reviews, vol. 37, no. 2, pp. 143-150, 2011.

[16] J. Von Pawel, J. H. Schiller, F. A. Shepherd et al., "Topotecan versus cyclophosphamide, doxorubicin, and vincristine for the treatment of recurrent small-cell lung cancer," Journal of Clinical Oncology, vol. 17, no. 2, pp. 658-667, 1999.

[17] M. E. R. O’Brien, T.-E. Ciuleanu, H. Tsekov et al., "Phase III trial comparing supportive care alone with supportive care with oral topotecan in patients with relapsed small-cell lung cancer," Journal of Clinical Oncology, vol. 24, no. 34, pp. 5441-5447, 2006.

[18] I. K. Demedts, K. Y. Vermaelen, and J. P. Van Meerbeeck, "Treatment of extensive-stage small cell lung carcinoma: current status and future prospects," European Respiratory Journal, vol. 35, no. 1, pp. 202-215, 2010.

[19] J.-H. Lee, J.-M. Lee, J.-K. Kim et al., "Antitumor activity of 7-[2(N-Isopropylamino)ethyl]-(20S)-camptothecin, CKD602, as a potent DNA topoisomerase I inhibitor," Archives of Pharmacal Research, vol. 21, no. 5, pp. 581-590, 1998.

[20] D. H. L. S. Yoon, C. Choi, J. C. Lee, J. S. Lee, and S. Kim, "Randomized phase II study of belotecan or topotecan chemotherapy as second-line chemotherapy after platinum-based first-line chemotherapy for small cell lung cancer," Annals of Oncology, vol. 25, p. iv515, 2014.

[21] K. H. Ban, T. O. Kim, J. Y. You et al., "A Randomized Prospective Multicenter Trial of Belotecan Versus Topotecan in Relapsed Small Cell Lung Cancer After First-Line Combination Therapy with Etoposide and Platinum," presented at the Korean Academy of Tuberculosis and Respiratory Diseases Abstract book, 2011, http://210.101.116.28/W_files/ftp43/0y909282_pv.pdf, http://m.riss.kr/search/detail/DetailView.do?p_mat type $=1 \mathrm{a} 0202 \mathrm{e} 37 \mathrm{~d} 52 \mathrm{c} 72 \mathrm{~d} \&$ control_no=79fb66efbe8a5cdlffe0bdc3ef48d419. 
[22] S. J. Kim, J. S. Kim, S. C. Kim et al., "A multicenter phase II study of belotecan, new camptothecin analogue, in patients with previously untreated extensive stage disease small cell lung cancer," Lung Cancer, vol. 68, no. 3, pp. 446-449, 2010.

[23] J. H. Schiller, K. Kim, P. Hutson et al., "Phase II study of topotecan in patients with extensive-stage small- cell carcinoma of the lung: An Eastern Cooperative Oncology Group trial," Journal of Clinical Oncology, vol. 14, no. 8, pp. 2345-2352, 1996.

[24] H. S. Kim, N. H. Park, S. Kang et al., "Comparison of the efficacy between topotecan- and belotecan-, a new camptothecin analog, based chemotherapies for recurrent epithelial ovarian cancer: a single institutional experience," Journal of Obstetrics and Gynaecology Research, vol. 36, no. 1, pp. 86-93, 2010. 


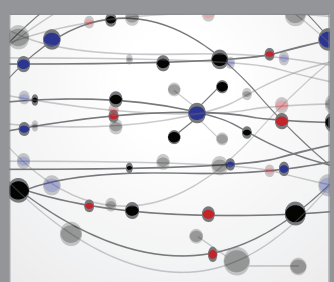

The Scientific World Journal
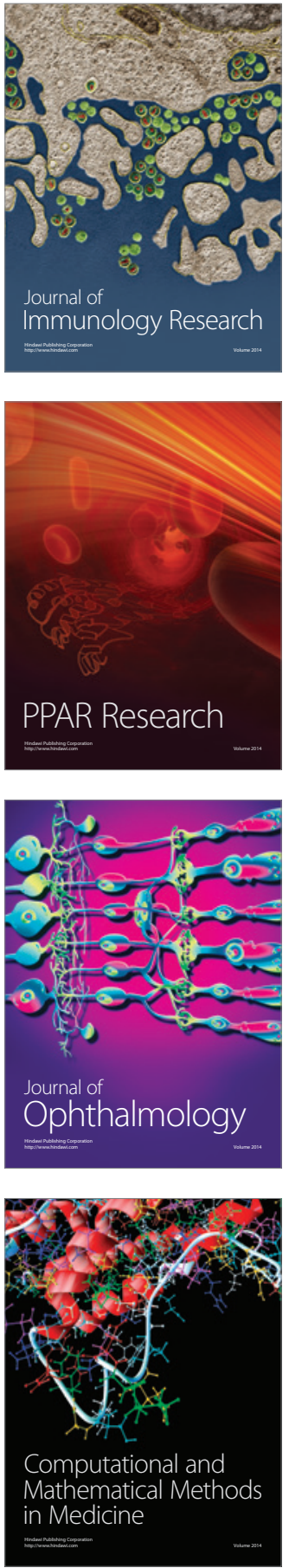

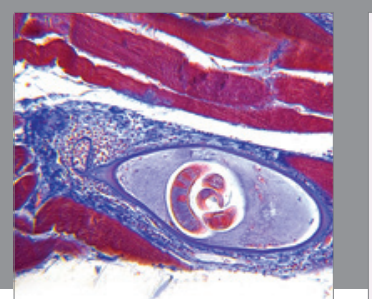

Gastroenterology Research and Practice

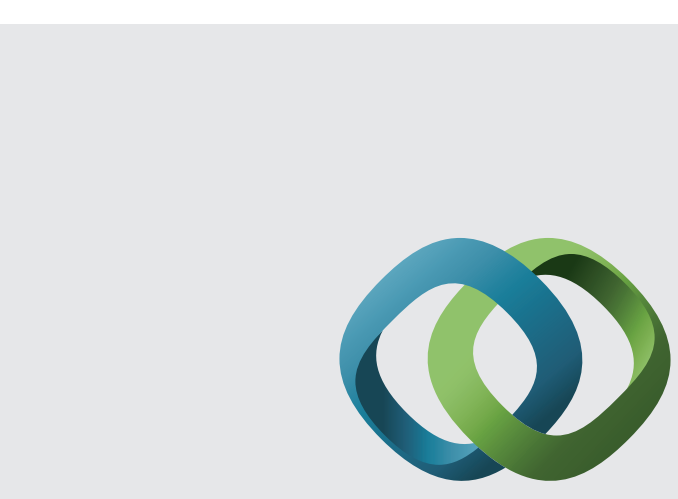

\section{Hindawi}

Submit your manuscripts at

http://www.hindawi.com
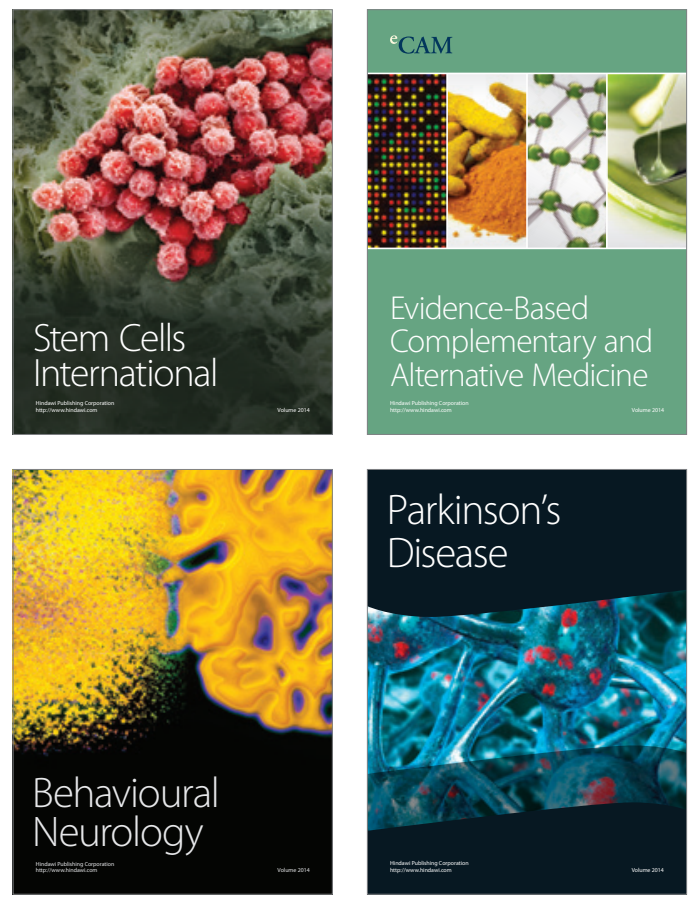
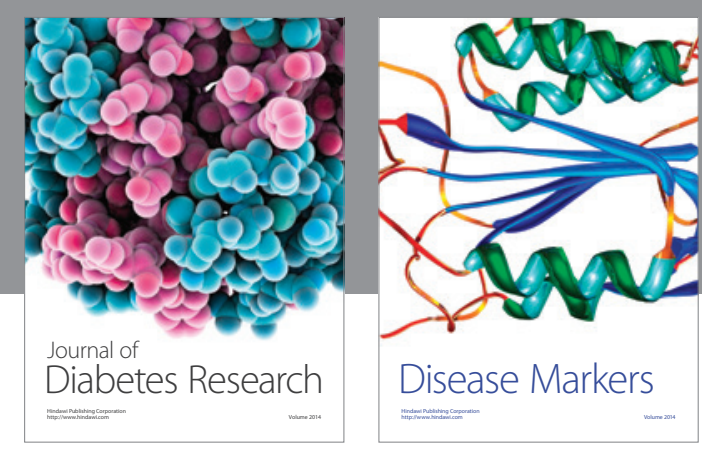

Disease Markers
